\section{Alterações no padrão alimentar de adolescentes com adequação pôndero-estatural e elevado percentual de gordura corporal}

\section{Alterations in the feeding patterns of adolescents with weight-height adequacy and high body fat percentage}

Valéria Cristina Ribeiro Vieira 1

Sílvia Eloiza Priore 2

Sônia M. R. Ribeiro 3

Sylvia do C. C. Franceschini 4

1 Departamento de Nutrição. Escola de Farmácia e Odontologia de Alfenas. Centro Universitário Federal. Rua Gabriel Monteiro da Silva, 714. Centro. Alfenas, MG, Brasil. CEP: 37.130-000. E-mail: valnut@ibest.com.br; valnut@vicosa.ufv.br 2-4 Departamento de Nutrição e Saúde. Universidade Federal de Viçosa, MG, Brasil.

\begin{abstract}
Objectives: alterations in the feeding patterns of adolescents as related to weight-height adequacy and high body fat percentage (\%BF).

Methods: a cross-sectional study was carried out with 99 adolescents, 70\% females. The body mass index was applied to diagnose weight-height adequacy, according to the cut-off point established by the World Health Organization. \%BF was obtained by the sum of skinfolds, according to Durnin and Rahaman, considering the values $>25 \%$ (females) and $>20 \%$ (males) high. A questionnaire was applied to investigate the feeding pattern.

Results: the median of daily meals was four Dinner was the most skipped main meal, however, the majority replaced it by a snack. Skipping dinner and having a snack in the afternoon were more common among girls $(p<0.01)$. About 11\% skipped breakfast. In the last years, meal frequency and food intake, except for vegetables, were reduced by the majority. On weekends, about $81 \%$ and $31 \%$ of the adolescents, respectively, modified the fat and sugar class and the vegetable class intake, consuming more sweets, fat foods and less vegetables. The most rejected food class consisted of vegetable. About $40.5 \%$ used diet/light food.

Conclusions: despite the weight-height adequacy and apparent concern related to weight control, these adolescents are under nutritional risk because of the high $\% B F$ and important feeding inadequacies.
\end{abstract}

Key words Adolescent, Anthropometry, Feeding

\section{Resumo}

Objetivos: avaliar o padrão alimentar de adolescentes com adequação pôndero-estatural e elevado percentual de gordura corporal (\%GC).

Métodos: realizou-se um estudo transversal com 99 adolescentes, sendo $70 \%$ do sexo feminino. Utilizou-se o indice de massa corporal para diagnosticar adequação pôndero-estatural, segundo a World Health Organization. Obteve-se o \%GC pelo somatório das pregas cutâneas, conforme Durnin e Rahaman, considerando elevados os valores $>25 \%$ (sexo feminino) e $>20 \%$ (masculino). Aplicou-se um questionário para investigação do hábito alimentar.

Resultados: a mediana de refeições diárias era quatro. O jantar era a refeição mais omitida, entretanto, a maioria o substituía por lanche. Omissão do jantar e realização do lanche da tarde foram mais freqüentes entre as adolescentes ( $p<0,01)$. Aproximadamente $11 \%$ omitiam o desjejum. Fracionamento da dieta e ingestão alimentares, exceto de hortaliças, foram reduzidos pela maioria, nos últimos anos. Cerca de $81 \%$ e $31 \%$, respectivamente, mencionaram alterar a ingestão do grupo das gorduras/doces e o das hortaliças, nos finais de semana, consumindo mais alimentos doces $e$ gordurosos e menos vegetais. O grupo mais rejeitado era o das hortaliças. Aproximadamente 40,5\% utilizavam alimentos diet/light.

Conclusões: apesar da adequação pôndero-estatural e da aparente preocupação em controlar o peso, esses adolescentes estão sob risco nutricional, devido ao elevado \%GC e aos importantes erros alimentares.

Palavras-chave Adolescente, Antropometria, Alimentação 


\section{Introdução}

Há alguns anos vem aumentando o interesse da Organização Mundial de Saúde e de outras instituições na saúde da população adolescente (faixa etária entre 10 e 20 anos). Nessa fase é importante a oferta correta de energia e de todos os nutrientes para suprir as necessidades aumentadas em função das mudanças fisiológicas. Entretanto, durante a adolescência é comum a realização de dietas inadequadas, muitas vezes autoprescritas e sem nenhum acompanhamento profissional, o que potencializa os riscos à saúde. ${ }^{1}$ Além disso, o estilo de vida, as tendências alimentares do mesmo grupo etário, de amigos e da própria família, contribuem para que, nem sempre, o adolescente tenha uma boa nutrição. ${ }^{2}$

Um estudo com adolescentes recém-ingressos na universidade, 3 encontrou cerca de $93 \%$ eutróficos segundo o índice de massa corporal (IMC), mas em torno de $63 \%$ desses apresentavam percentual de gordura acima do esperado. Em relação aos hábitos alimentares, observou-se que $57,3 \%$ dos entrevistados omitiam uma das refeições principais, sendo que $31,7 \%$ não realizavam o desjejum. O grupo das hortaliças foi o mais rejeitado, sendo que mais de $28 \%$ dos estudados ingeriam quatro vezes ou menos, por semana, alimentos desse grupo. As frutas eram ingeridas com essa freqüência por mais de $75 \%$ dos estudados, sendo que aproximadamente $35 \%$ ingeriam uma vez ou menos. Por outro lado, $37 \%$ ingeriam alimentos doces ou gordurosos todos os dias. Esses resultados, oriundos de um questionário acerca do consumo semanal dos grupos de alimentos, chamaram a atenção, pois demonstram falhas importantes na alimentação dos adolescentes, como baixa ingestão de fontes de vitaminas, minerais e fibras e consumo acentuado de açúcares e gorduras.

O aumento do percentual de obesos adultos 4,5 reforça a necessidade de avaliar os adolescentes, visto que o controle de doenças crônico-degenerativas deve ser iniciado em fases precoces da vida. A definição de fatores ambientais, inclusive hábitos alimentares, relacionados à gênese da obesidade, pode ajudar a melhor identificar subgrupos de crianças e adolescentes com riscos aumentados para doenças e aumentar a efetividade das medidas preventivas. 6

Fatores relacionados à gordura corporal, especialmente em indivíduos eutróficos, ainda são um tópico de muito estudo. Sobretudo em adolescentes, os trabalhos nessa área são restritos e a escassez de conhecimento dificulta intervenções corretas, entre elas as dietéticas.

Tais observações apontaram para a necessidade de análises mais detalhadas do hábito alimentar de adolescentes eutróficos, bem como de um conhecimento mais apurado de sua composição corporal. $\mathrm{O}$ objetivo principal do presente estudo foi, portanto, avaliar aspectos do padrão alimentar de adolescentes com adequação pôndero-estatural e elevado percentual de gordura corporal.

\section{Métodos}

Participaram do estudo 99 adolescentes, sendo $69,7 \%$ do sexo feminino. A maioria (90,9\%) tinha 14 anos ou mais, sendo que $45,5 \%$ se encontravam na adolescência final, ou seja, entre 17 e 19 anos. A maior parte $(55,5 \%)$ foi entrevistada durante o atendimento em um programa de atenção à saúde do adolescente. Tal programa objetiva orientar nutricionalmente adolescentes filhos de servidores de uma universidade pública e estudantes do colégio universitário (ensino médio) ou dos cursos de graduação dessa mesma universidade, em Viçosa, Minas Gerais, Brasil. Os estudantes procuraram espontaneamente pelo atendimento. Todas as informações utilizadas na pesquisa foram fornecidas pelos adolescentes em sua primeira consulta, para evitar que as respostas fossem influenciadas pelas orientações recebidas. Outra parcela das entrevistas $(35,4 \%)$ foi realizada no próprio colégio universitário e $9,1 \%$ delas nas residências de graduandos recém-ingressos na universidade.

O estudo foi do tipo transversal, com o intuito de delinear o padrão alimentar do grupo específico de adolescentes estudados para posterior formulação de hipóteses, a serem testadas em amostras representativas. Não houve nesse estudo, portanto, a pretensão de estimar prevalências, representar determinada população ou propiciar a extrapolação dos resultados obtidos.

Os adolescentes foram avaliados, inicialmente, em relação à adequação pôndero-estatural por meio do índice de massa corporal. Os adequados, segundo a Organização Mundial de Saúde, 7 foram então avaliados em relação ao percentual de gordura corporal (\%GC).

Obteve-se o peso com balança eletrônica Kratos ${ }^{\circledR}$, com capacidade de 150 kg e subdivisão de 50 gramas. A estatura foi obtida com fita métrica metálica, com extensão de 2,0 metros, dividida em centímetros, subdividida em milímetros e com esquadro plástico acoplado à sua extremidade. As técnicas utilizadas para a obtenção do peso e da estatura foram as preconizadas por Jelliffe. 8

$\mathrm{O} \% \mathrm{GC}$ foi obtido pelo somatório de quatro 
pregas cutâneas (tricipital, bicipital, subescapular e suprailíaca), segundo o proposto por Durnin e Rahaman ${ }^{9}$. Considerou-se \%GC elevado valores acima de $25 \%$ e de $20 \%$ para os sexos feminino e masculino, respectivamente. 10

As medidas das pregas foram obtidas utilizandose o equipamento Lange Skinfold Caliper, sendo que cada uma foi verificada três vezes, ${ }^{11}$ trabalhando-se com a média dos dois valores mais próximos ou dos dois maiores quando os três valores eram consecutivos.

Características do padrão alimentar foram obtidas por meio de entrevista, tendo sido avaliados os seguintes aspectos: número de refeições realizadas e omissão de alguma das principais (desjejum, almoço e jantar), nos dias úteis; alterações na alimentação nos finais de semana e as mudanças de hábito alimentar nos últimos três anos, considerando-se o fracionamento da dieta, o volume das refeições e a ingestão dos grupos de alimentos da Pirâmide Alimentar (cereais, hortaliças, frutas,carnes/ovos/ leguminosas, laticínios e gorduras/doces).12 Considerou-se esse período de três anos, para avaliação de alterações na ingestão alimentar, visando enfocar a fase de entrada na adolescência, tendo em vista suas repercussões no comportamento alimentar. 13 A alteração no hábito alimentar (redução ou aumento) foi definida pelos próprios adolescentes, ou seja, eles responderam diretamente à pergunta - "Houve alteração? Caso tenha havido, aumentou ou reduziu?". Não foram, portanto, estabelecidos pelos pesquisadores quaisquer pontos de corte para caracterizar as alterações, sendo as análises baseadas unicamente no referido pelos adolescentes. Questionou-se ainda, na entrevista, sobre rejeições alimentares e utilização de alimentos diet/light.

Os dados foram armazenados e analisados utilizando-se o software Epi-info. Foi aplicado o teste estatístico do qui-quadrado entre o número de refeições realizadas nos dias úteis de acordo com o sexo e a omissão de refeições nos dias úteis de acordo com o sexo.

O nível de rejeição fixado foi igual ou menor do que 0,05 .

A participação dos adolescentes nesse estudo foi espontânea, após terem sido esclarecidos sobre os objetivos do mesmo. Aqueles que apresentaram algum problema de saúde ou nutricional, identificado durante a entrevista no colégio ou residência, foram encaminhados ao atendimento individualizado no programa de atenção à saúde do adolescente desenvolvido na universidade.

\section{Resultados}

A mediana do número de refeições diárias realizadas pelos adolescentes foi quatro, valor adotado para categorizar essa variável, conforme apresentado na Tabela 1. Verificou-se que o almoço não foi omitido por nenhum dos estudados. A realização do lanche da tarde era significantemente mais comum entre as adolescentes $(p=0,009)$. Obteve-se diferença significante entre os sexos também em relação à omissão do jantar $(p=0,003)$, que era cerca de três vezes mais prevalente entre as adolescentes. (Tabela 2) Verificou-se que $89,5 \%$ dos que não realizavam o jantar tradicional substituíam-no por um lanche ou refeição mais leve. Os que não jantavam nem lanchavam no horário característico do jantar eram todos do sexo feminino. (Tabela 3 )

Maior percentual $(89,5 \%)$ das adolescentes que jantavam tinha o hábito de merendar à tarde quando comparadas com as que não jantavam (78\%). (Tabela 4)

Verificou-se que $63,6 \%$ dos que não realizavam o desjejum também não faziam colação, ou seja, o almoço tornava-se seu verdadeiro desjejum.(Tabela 5) Além disso, $54,5 \%$ dos que omitiam o desjejum também não faziam a ceia, prolongando seu período de jejum. (Tabela 6)

Dos adolescentes que responderam a questão sobre alterações alimentares nos últimos três anos, $75 \%$ haviam modificado pelo menos um dos aspectos do seu hábito alimentar, listados na questão (fracionamento da dieta, volume das refeições, ingestão dos grupos de alimentos) e $92 \%$ alteravam algum desses aspectos nos finais de semana. (Tabela 7)

O fracionamento da dieta e o volume das refeições eram alterados nos finais de semana por $61,5 \%$ e $47,9 \%$ dos adolescentes estudados, respectivamente. (Tabela 7) A maioria passava a comer mais vezes ao dia e volumes maiores, além de aumentar o consumo de todos os grupos de alimentos, exceto hortaliças. Mais de $80 \%$ dos estudados informaram alterar a ingestão de gorduras/doces nesses dias, sendo que $91 \%$ desses aumentavam-na. A ingestão de hortaliças era modificada por menor parcela dos adolescentes $(31,3 \%)$, mas, essa alteração significava redução para $80 \%$ desses. Nos últimos três anos, o aspecto mais variado foi também a ingestão de gorduras/doces, seguido do volume das refeições e fracionamento da dieta. Com exceção do grupo das hortaliças, houve redução no consumo de todos os grupos de alimentos para a maior parte dos adolescentes. 
Na Tabela 8 verifica-se que quase $80 \%$ dos entrevistados relataram ter rejeição por algum alimento, sendo o grupo alimentar mais rejeitado o das hortaliças. Cerca de 15\%, quando questionados sobre sua rejeição às hortaliças, citaram apenas uma, enquanto que $12 \%$ disseram que não comiam verduras ou legumes em geral e $11 \%$ admitiram ser adversos a qualquer tipo de hortaliça.

Tabela 1

Número de refeições realizadas nos dias úteis de acordo com o sexo.

\begin{tabular}{|c|c|c|c|c|c|c|}
\hline \multirow{3}{*}{ Número de refeições/dia } & \multicolumn{4}{|c|}{ Sexo } & & \\
\hline & \multicolumn{2}{|c|}{ Masculino } & \multicolumn{2}{|c|}{ Feminino } & \multicolumn{2}{|c|}{ Total } \\
\hline & $\mathrm{n}$ & $\%$ & $\mathrm{n}$ & $\%$ & $\mathrm{n}$ & $\%$ \\
\hline$<4$ & 18 & 60,0 & 32 & 46,4 & 50 & 50,5 \\
\hline$>4$ & 12 & 40,0 & 37 & 53,6 & 49 & 49,5 \\
\hline Total & 30 & 100,0 & 69 & 100,0 & 99 & 100,0 \\
\hline
\end{tabular}

Teste do qui-quadrado: $p=0,21$

Tabela 2

Omissão de refeições nos dias úteis de acordo com o sexo.

\begin{tabular}{|c|c|c|c|c|c|c|}
\hline \multirow{3}{*}{ Refeições omitidas } & \multicolumn{4}{|c|}{ Sexo } & & \\
\hline & \multicolumn{2}{|c|}{ Masculino } & \multicolumn{2}{|c|}{ Feminino } & \multicolumn{2}{|c|}{ Total } \\
\hline & $\mathrm{n}$ & $\%$ & $\mathrm{n}$ & $\%$ & $\mathrm{n}$ & $\%$ \\
\hline Desjejum & 4 & 13,3 & 7 & 10,1 & 11 & 11,1 \\
\hline Colação & 22 & 73,3 & 36 & 52,2 & 58 & 58,6 \\
\hline Lanche (1) & 14 & 46,7 * & 13 & 18,8 & 27 & 27,3 \\
\hline Jantar (2) & 7 & 23,3 & 50 & 72,5 ** & 57 & 57,6 \\
\hline Ceia & 16 & 53,3 & 40 & 58,0 & 56 & 56,6 \\
\hline
\end{tabular}

(1) Considerou-se como "lanche" a refeição realizada no meio da tarde; (2) Considerou-se como "jantar" a refeição similar ao almoço, ou seja, contendo alimentos comumente presentes no típico almoço do brasileiro; Teste do qui-quadrado: ${ }^{*} p=0,009 ;{ }^{*} p=0,003$ 
Realização de lanche em substituição ao jantar, de acordo com o sexo.

\begin{tabular}{|c|c|c|c|c|c|c|}
\hline \multirow{3}{*}{$\begin{array}{l}\text { Realização de lanche em } \\
\text { substituição ao jantar }\end{array}$} & \multicolumn{4}{|c|}{ Sexo } & \multirow{2}{*}{\multicolumn{2}{|c|}{ Total }} \\
\hline & \multicolumn{2}{|c|}{ Masculino } & \multicolumn{2}{|c|}{ Feminino } & & \\
\hline & $n$ & $\%$ & $n$ & $\%$ & $\mathrm{n}$ & $\%$ \\
\hline Sim & 7 & 100,0 & 44 & 88,0 & 51 & 89,5 \\
\hline Não & - & - & 6 & 12,0 & 6 & 10,5 \\
\hline Total & 7 & 100,0 & 50 & 100,0 & 57 & 100,0 \\
\hline
\end{tabular}

\section{Tabela 4}

Realização do lanche da tarde de acordo com a realização do jantar, entre as adolescentes do sexo feminino.

\begin{tabular}{|c|c|c|c|c|c|c|}
\hline \multirow{3}{*}{ Realização do jantar } & \multicolumn{4}{|c|}{ Realização do lanche da tarde } & & \\
\hline & \multicolumn{2}{|c|}{$\operatorname{Sim}$} & \multicolumn{2}{|c|}{ Não } & \multicolumn{2}{|c|}{ Total } \\
\hline & $\mathrm{n}$ & $\%$ & $\mathrm{n}$ & $\%$ & $\mathrm{n}$ & $\%$ \\
\hline Sim & 17 & 89,5 & 2 & 10,5 & 19 & 100,0 \\
\hline Não & 39 & 78,5 & 11 & 22,0 & 50 & 100,0 \\
\hline Total & 56 & 81,2 & 13 & 18,8 & 69 & 100,0 \\
\hline
\end{tabular}

Tabela 5

Realização da colação, de acordo com a realização do desjejum

\begin{tabular}{|c|c|c|c|c|c|c|}
\hline \multirow{3}{*}{ Realização do desjejum } & \multicolumn{4}{|c|}{ Realização da colação } & & \\
\hline & \multicolumn{2}{|c|}{$\operatorname{sim}$} & \multicolumn{2}{|c|}{ Não } & \multicolumn{2}{|c|}{ Total } \\
\hline & $\mathrm{n}$ & $\%$ & $\mathrm{n}$ & $\%$ & $\mathrm{n}$ & $\%$ \\
\hline Sim & 37 & 42,1 & 51 & 57,9 & 88 & 100,0 \\
\hline Não & 4 & 36,4 & 7 & 63,6 & 11 & 100,0 \\
\hline Total & 41 & 41,4 & 58 & 58,6 & 99 & 100,0 \\
\hline
\end{tabular}


Realização da ceia, de acordo com a realização do desjejum.

\begin{tabular}{|c|c|c|c|c|c|c|}
\hline \multirow{3}{*}{ Realização do desjejum } & \multicolumn{4}{|c|}{ Realização da colação } & & \\
\hline & \multicolumn{2}{|c|}{ Sim } & \multicolumn{2}{|c|}{ Não } & \multicolumn{2}{|c|}{ Total } \\
\hline & $\mathrm{n}$ & $\%$ & $\mathrm{n}$ & $\%$ & $\mathrm{n}$ & $\%$ \\
\hline Sim & 51 & 57,9 & 37 & 42,1 & 88 & 100,0 \\
\hline Não & 5 & 45,5 & 6 & 54,5 & 11 & 100,0 \\
\hline Total & 56 & 56,6 & 43 & 43,4 & 99 & 100,0 \\
\hline
\end{tabular}

Dos adolescentes que responderam a questão sobre alterações alimentares nos últimos três anos, $75 \%$ haviam modificado pelo menos um dos aspectos do seu hábito alimentar, listados na questão (fracionamento da dieta, volume das refeições, ingestão dos grupos de alimentos) e $92 \%$ alteravam algum desses aspectos nos finais de semana. (Tabela 7)

$O$ fracionamento da dieta e o volume das refeições eram alterados nos finais de semana por $61,5 \%$ e $47,9 \%$ dos adolescentes estudados, respecti- vamente. (Tabela 7) A maioria passava a comer mais vezes ao dia e volumes maiores, além de aumentar o consumo de todos os grupos de alimentos, exceto hortaliças. Mais de $80 \%$ disseram alterar a ingestão de gorduras/doces nesses dias, sendo que $91 \%$ desses aumentavam-na. A ingestão de hortaliças era modificada por menor parcela dos adolescentes $(31,3 \%)$, mas, essa alteração significava redução para $80 \%$ desses.

Tabela 7

Alterações da freqüência alimentar.

\begin{tabular}{|c|c|c|c|c|c|c|c|c|c|c|c|c|c|c|c|c|}
\hline \multirow{4}{*}{ Variáveis } & \multicolumn{8}{|c|}{ Alterações nos finais de semana } & \multicolumn{8}{|c|}{ Alterações nos últimos três anos } \\
\hline & \multicolumn{8}{|c|}{ Tipo de alteração } & \multicolumn{8}{|c|}{ Tipo de alteração } \\
\hline & \multicolumn{2}{|c|}{ Sim } & \multicolumn{2}{|c|}{ Não } & \multicolumn{2}{|c|}{ Aumentou } & \multicolumn{2}{|c|}{ Reduziu } & \multicolumn{2}{|c|}{ Sim } & \multicolumn{2}{|c|}{ Não } & \multicolumn{2}{|c|}{ Aumentou } & \multicolumn{2}{|c|}{ Reduziu } \\
\hline & $\mathrm{n}$ & $\% \#$ & $\mathrm{n}$ & $\% \#$ & $\mathrm{n}$ & $\%$ * & $\mathrm{n}$ & $\%$ * & $\mathrm{n}$ & $\% \#$ & $\mathrm{n}$ & $\% \#$ & $\mathrm{n}$ & $\%$ * & $\mathrm{n}$ & $\%$ * \\
\hline Alteração em pelo menos um aspecto & 88 & 91,7 & 8 & 8,3 & - & - & - & - & 72 & 75,0 & 24 & 25,0 & - & - & - & - \\
\hline Fracionamento da dieta & 59 & 61,5 & 37 & 38,5 & 31 & 52,5 & 28 & 47,5 & 47 & 49,0 & 49 & 51,0 & 16 & 34,0 & 31 & 66,0 \\
\hline Volume de cada refeição & 46 & 47,9 & 50 & 52,1 & 29 & 63,0 & 17 & 37,0 & 51 & 53,1 & 45 & 46,9 & 28 & 54,9 & 23 & 45,1 \\
\hline Ingestão de cereais & 34 & 35,4 & 62 & 64,6 & 34 & 100,0 & - & - & 16 & 16,6 & 80 & 83,4 & 7 & 43,8 & 9 & 56,2 \\
\hline Ingestão de gorduras/doces & 78 & 81,3 & 18 & 18,7 & 71 & 91,0 & 7 & 9,0 & 52 & 54,2 & 44 & 45,8 & 17 & 32,7 & 35 & 67,3 \\
\hline Ingestão de carnes/ovos/leguminosas & 26 & 27,1 & 70 & 72,9 & 21 & 80,8 & 5 & 19,2 & 24 & 25,0 & 72 & 75,0 & 7 & 29,2 & 17 & 70,8 \\
\hline Ingestão de leite e derivados & 17 & 17,7 & 79 & 82,3 & 11 & 64,7 & 6 & 35,3 & 29 & 30,2 & 67 & 69,8 & 11 & 37,9 & 18 & 62,1 \\
\hline Ingestão de hortaliças & 30 & 31,3 & 66 & 68,7 & 6 & 20,0 & 24 & 80,0 & 33 & 34,4 & 63 & 65,6 & 23 & 69,7 & 10 & 30,3 \\
\hline Ingestão de frutas & 28 & 29,2 & 68 & 70,8 & 19 & 67,9 & 9 & 32,1 & 37 & 38,5 & 59 & 61,5 & 16 & 43,2 & 21 & 56,8 \\
\hline
\end{tabular}

\# Calculado sobre o total de 96 adolescentes que responderam a essa questão; * calculado sobre o total dos que alteraram cada variável 
Nos últimos três anos, o aspecto mais variado foi também a ingestão de gorduras/doces, seguido do volume das refeições e fracionamento da dieta. Com exceção do grupo das hortaliças, houve redução no consumo de todos os grupos de alimentos para a maior parte dos adolescentes.

Na Tabela 8 observa-se que quase $80 \%$ dos entrevistados relataram ter rejeição por algum alimento, sendo o grupo alimentar mais rejeitado o das hortaliças. Cerca de $15 \%$, quando questionados sobre sua rejeição às hortaliças, citaram apenas uma, enquanto que $12 \%$ disseram que não comiam verduras ou legumes em geral e $11 \%$ admitiram ser adversos a qualquer tipo de hortaliça.

\section{Discussão}

Vários fatores podem estar influenciando o comportamento apresentado em relação ao fracionamento da dieta. Segundo Tojo et al.,14 o número de refeições que os mais jovens realizam dá-se muito por influência das tradições, do nível socioeconômico e educacional da família, da influência dos colegas e até da mídia. As implicações de um fracionamento inadequado da dieta e de um descontrole de horários para se alimentar podem incluir problemas decorrentes do jejum prolongado, como maior propensão à gastrite ou realização de refeições excessivamente volumosas para "compensar" o jejum, situação que pode favorecer sintomas como a dispepsia e a distensão gástrica. ${ }^{15}$

Além disso, o fracionamento da dieta está entre os fatores que podem influenciar no metabolismo de carboidratos e gorduras e, conseqüentemente, na composição corporal.16 Estando todos os adolescentes estudados com excesso de gordura corporal, essa questão talvez seja um dos agravantes da elevação. Assim, é importante analisar a realização rotineira de refeições, bem como a variação de comportamento de acordo com o sexo e com as situações de vida específicas, buscando, inclusive, as motivações que levam os adolescentes a se alimentarem mais ou menos vezes ao longo do dia.

A diferença entre os sexos em relação à omissão do jantar também foi encontrada em um estudo com adolescentes de 12 a 18 anos no município de São Paulo. 17 Essa tendência de restringir a alimentação no período noturno pode indicar uma maior preocupação com o controle do peso, sobretudo entre as adolescentes, uma vez que, segundo o senso comum, deve-se evitar refeições "pesadas" à noite para não
Tabela 8

Rejeições alimentares e utilização de alimentos diet/light.

\begin{tabular}{lcc}
\hline Comportamento alimentar & $\mathbf{n}$ & $\%$ \\
\hline Rejeitam algum alimento & 78 & 78,8 \\
Hortaliças & 64 & 64,6 \\
Carnes/ovos/leguminosas & 20 & 20,2 \\
Gorduras/doces & 18 & 18,2 \\
Frutas & 16 & 16,2 \\
Cereais & 7 & 7,07 \\
Leite e derivados & 4 & 4,04 \\
Utilizam algum alimento diet ou light & 40 & 40,4 \\
\hline
\end{tabular}

engordar. Cavadini 18 atribui a omissão de refeições entre as adolescentes a uma possível motivação para a perda de peso, de tal forma que, a falta de apetite por vezes referida poderia ser, talvez, uma estratégia para não engordar ou para emagrecer.

Questionou-se se a realização do lanche da tarde, significantemente mais comum entre as adolescentes, poderia estar interferindo na realização do jantar. Porém, como maior percentual das adolescentes que jantava tinha o hábito de merendar à tarde quando comparadas com as que não jantavam, não se pode dizer que o fato das adolescentes realizarem mais o lanche da tarde determinasse que omitissem mais o jantar. As razões para essas diferenças entre os sexos parecem ser, de fato, culturais e comportamentais. 18

Os resultados de um estudo realizado com adolescentes com idade média de 14 anos, 19 também remetem à hipótese de que o lanche está tomando o lugar do jantar tradicional. Outro estudo ${ }^{3}$ com adolescentes universitários, em que $49 \%$ dos estudados preferiam lanchar no horário característico do jantar a realizar o jantar tradicional, (mesmo essa refeição sendo oferecida a preço subsidiado no restaurante universitário) mostra tendência similar. Dessa forma, talvez fosse mais efetivo estimular entre esses adolescentes a realização de refeições balanceadas e melhor distribuídas ao longo do dia, prioritariamente à designação do tipo de refeição, uma vez que lanches também podem ser nutritivos se os alimentos forem combinados adequadamente.

Estudando a realização de refeições por estudantes adolescentes com idade média de 14 anos, Gambardella et al. 19 observaram que $56 \%$ dos que não tomavam o desjejum também não realizavam ceia ou colação, permanecendo em jejum do jantar 
do dia anterior ao almoço do dia seguinte. Observaram também que, dos demais que não faziam o desjejum, 33\% consumiam colação e $11 \%$ apontaram que ceiavam; percentuais, portanto, inferiores aos do presente estudo $(36,4$ e $45,5 \%$, respectivamente). Gambardella et al. ${ }^{19}$ assinalam que a prática de omitir o desjejum inviabiliza a elevação da glicemia, necessária às atividades matinais e favorece uma possível deficiência de cálcio, uma vez que essa refeição geralmente concentra o maior consumo diário de alimentos fontes desse mineral.

Considerando-se as variações intraindividuais no hábito alimentar características desse grupo etário, bem como o próprio início da adolescência um marco de mudanças no comportamento, inclusive o alimentar, 13 procurou-se conhecer as variações oriundas do ingresso na adolescência ou relacionadas a oscilações comportamentais próprias da fase. Os resultados mostrados confirmam o quanto são comuns essas alterações.

Os resultados acerca das modificações alimentares nos finais de semana permitem supor o desbalanceamento da dieta nesses dias, condição que, se periodicamente repetida, potencializará os riscos nutricionais do grupo. 13 Nesse sentido, devese buscar alternativas de incluir vegetais nas preparações típicas de final de semana e incentivar hábitos corretos verificados, como o aumento no consumo de frutas.

$\mathrm{O}$ aumento do volume ingerido nos últimos anos pode ser justificado pelo aumento das necessidades nutricionais na adolescência, mas também pode ser conseqüência do menor número de refeições diárias, já que $66 \%$ dos que mudaram o fracionamento, reduziram-no. A redução no consumo de todos os grupos de alimentos, exceto hortaliças, pode estar ligada ao ingresso na adolescência, quando os incrementos normais da fase talvez levem o indivíduo a pensar que deva controlar o peso, restringindo o número de refeições diárias e os alimentos considerados "engordativos". Talvez visando controle do peso e baseando-se em pré-concepções a respeito de dietas de emagrecimento, 20 muitos adolescentes tenham reduzido a ingestão de cereais, gorduras/ doces, carnes/ovos/leguminosas e aumentado a ingestão de hortaliças. A ingestão de leite e derivados também pode ter sido reduzida por esse motivo ou pelo estigma do leite como um "alimento de criança", "desnecessário aos já crescidos". A literatura refere que esse alimento é muitas vezes considerado próprio da infância por parte dos adolescentes, que passam a restringir sua ingestão e preferir outras bebidas. 21,22

A redução indiscriminada no consumo dos grupos de alimentos base da alimentação, como os cereais, ou fonte de importantes nutrientes, como o leite, as carnes e as frutas, é contra-indicada para qualquer parcela da população, sobretudo para os adolescentes que estão em fase de intenso crescimento e desenvolvimento. ${ }^{14}$ Reforça-se, assim, a necessidade de medidas educativas específicas para esse grupo etário, de maneira a transmitir informações corretas, desmistificando concepções à respeito da alimentação e alertando-os acerca dos riscos nutricionais por carência e excesso de nutrientes e energia.

Outro motivo de alerta é o fato dos adolescentes poderem estar equivocadamente acreditando em um suposto resultado de suas restrições alimentares, já que, apesar de adequados na relação peso/estatura, todos estavam com excesso de gordura corporal. Desse modo, muitos podem estar satisfeitos com o peso atual, mas podem ser futuros candidatos à obesidade ou sobrepeso devido à composição corporal apresentada, já que o aumento da massa gordurosa provoca queda no metabolismo basal e favorece o ganho de peso. 16,23

Essas observações revelam o quão preocupante é a conduta adotada pelos adolescentes estudados em relação à sua alimentação, pois, eles julgam estar procedendo corretamente ou, pelo menos, bem sucedidamente, atingindo seu objetivo de manter o "peso ideal"; no entanto, sua gordura corporal é elevada, podendo estar comprometida sua saúde atual e futura.

Estudos têm mostrado um aumento do risco para doenças cardiovasculares em populações cada vez mais jovens, estando entre esses riscos a hipercolesterolemia. ${ }^{24-26}$ Tojo et al. ${ }^{22}$ relatam que estudos epidemiológicos e de necrópsias em crianças e adolescentes evidenciam que a aterosclerose começa nessa idade. Na Espanha, especificamente, entre $10 \mathrm{e}$ $25 \%$ das crianças e adolescentes, segundo os autores citados, apresentam níveis elevados de colesterol.

As restrições alimentares, por outro lado, podem levar à deficiência de nutrientes importantes, como o ferro e o cálcio, expondo esses adolescentes a riscos atuais e futuros de doenças como a anemia e a osteoporose, respectivamente, e podendo dificultar seu crescimento e desenvolvimento normais. 18,27

As análises das informações prestadas pelos adolescentes a respeito das alterações alimentares nos últimos anos apresentam limitações no que tange à capacidade recordatória desses indivíduos, ou seja, é necessário levar em conta o grau de precisão em que eles lembraram, identificaram e expressaram a informação buscada. Poder-se-ia questionar a confiabilidade de tais informações considerando, também, 
a possibilidade de que, talvez, estivessem desejando transmitir a imagem de "bem informados" e "preocupados com a saúde",28 ao responderem a questão sobre as alterações alimentares nos últimos anos. Essa hipótese, contudo, parece improvável, já que tal intenção também teria estado presente (e assim, seria igualmente detectável) nas informações sobre as alterações nos finais de semana e sobre suas rejeições alimentares, o que não ocorreu. Além disso, muitos dos estudados eram atendidos no programa de atenção à saúde do adolescente e geralmente o procuravam por iniciativa própria, ou seja, demonstravam ter interesse, prestando informações exatas para poderem receber orientações adequadas.

Os resultados acerca das rejeições alimentares reforçam outros estudos realizados com adolescentes em que o grupo das hortaliças também é o mais rejeitado,3,18,29 e apontam para a necessidade de se desenvolverem formas alternativas de ingestão de vegetais (bolos, tortas, suflês, sucos, sopas, sanduíches, etc.), buscando-se a harmonia entre o paladar dos adolescentes e essas importantes fontes de vitaminas, minerais e fibras.

O fato de o grupo do leite e derivados ter sido o menos rejeitado, vai de encontro às modificações relatadas para os últimos anos, em que a maioria dos que alteraram a ingestão de laticínios, reduziram-na. Assim, pode-se conceber que, talvez, os adolescentes tenham reduzido o consumo de leite não por esse alimento ter-se tornado aversivo, mas, por terem passado a considerar o consumo inapropriado para eles, associando-o a uma dieta "para engordar", ou característica da infância. Como grande parte estava ingressando na adolescência nos últimos três anos, a negação das "coisas próprias de criança" e adesão à "alimentação de jovem" (preferindo refrigerantes, por exemplo) é um comportamento muitas vezes encontrado. 21,22-29

Chama a atenção o percentual de utilização de alimentos diet/light por uma população com peso adequado. No trabalho com adolescentes de 12 a 18 anos estudantes da rede pública do município de São Paulo17 encontrou-se um percentual de utilização desses alimentos inferior ao deste estudo $(21,9 \%)$. A análise desse aspecto confrontada com o conjunto dos resultados encontrados permite supor que a alimentação equilibrada está sendo negligenciada por esses adolescentes como a forma mais adequada e eficiente de conjugar saúde e peso desejável. Ao utilizarem produtos diet/light e, ao mesmo tempo, rejeitarem alimentos essenciais ao bom funcionamento do organismo, podem estar procurando uma estratégia mais cômoda e efetiva para atingir o ideal de estética e vitalidade.

Assim, apesar da eutrofia e da aparente preocupação com o controle do peso, eles estão sob risco nutricional, em virtude do elevado \%GC e dos importantes erros alimentares apresentados, os quais podem comprometer seu crescimento e desenvolvimento, bem como sua saúde atual e futura.

\section{Agradecimentos}

Ao Programa Interinstitucional de Bolsas de Iniciação Científica (PIBIC) do CNPq. 


\section{Referências}

1. Ferrarotti NG. La salud e las áreas de educación para la salud en la adolescencia. In: OMS (Organización Mundia de la Salud). La salud del adolescente e del joven en las Americas. Washington (DC); 1985. p. 3-14. (Publicación científica, 552).

2. Saito MI. Desnutrição. In: Coates V, Françoso LA, Beznos GW. Medicina do adolescente. São Paulo: Sarvier; 1993. p 59-65.

3. Vieira VCR, Priore SE, Ribeiro SMR, Franceschini SCC Almeida LPA. Perfil socioeconômico, de saúde e nutrição de adolescentes recém-ingressos em uma universidade pública brasileira. Rev Nutr 2002; 15: 273-82

4. Ministério da Saúde. Instituto Nacional de Alimentação e Nutrição. Pesquisa Nacional sobre Saúde e Nutrição: condições nutricionais da população brasileira: adulto e idoso. Brasília (DF); 1991.

5. Mondini L, Monteiro CA. Mudanças no padrão de alimentação. In: Monteiro CA. Velhos e novos males de saúde no Brasil: evolução do país e de suas doenças. 2. ed. São Paulo: Hucitec; 2000. p. 79-89.

6. Maffeis C, Talameni G, Tato L. Influence of diet, physical activity and parents'obesity on children's adiposity: a fouryear longitudinal study. Int J Obes 1998; 22: 758-64.

7. WHO (Word Health Organization). Physical status: the use and interpretation of anthropometry. Geneva; 1995

8. Jelliffe DB. Evaluación del estado de nutrición de la comunidad. Ginebra: OMS; 1968. (Série de Monografias, 53).

9. Durnin JVGA, Rahaman MM. The assessment of the amount of fat in the human body from measurements of skinfold thickness. Br J Nutr 1967; 21: 681-9.

10. Sigulem DM, Veiga VG, Priore SE. Obesidade em adolescentes de baixa renda. In: Fisberg M. Obesidade na infância e na adolescência. São Paulo: Fundo Editorial Byk; 1995. p. 80-3.

11. Blackburn GL, Thorton PA. Avaliação nutricional do paciente hospitalizado. In: Margen S, Caan B. Clínicas médicas da América do Norte. Rio de Janeiro: Interamericana; 1979. p. 1103-15

12. United States Department of Agriculture. Human Nutrition Information Service. The food guide pyramid. Hyattsville; 1992

13. Moreiras OE, Carbajal A. Determinantes socioculturales del comportamiento alimentario de los adolescentes. An Esp Pediatr 1992; 36: 102-5.

14. Tojo R, Leis R, Recarey MD, Pavon P. Hábitos alimentares das crianças em idade pré-escolar e escolar: riscos para a saúde e estratégias para a intervenção. In: Nestlé Nutrition Services. A alimentação da idade pré-escolar até a adolescência. São Paulo; 1995. p. 11-13. (Seminário Nestlé Nutrition, 37).
15. Mahan LK, Escott-Stump S. Cuidado nutricional nas doenças da cavidade oral, esôfago e estômago. In: Krause: alimentos, nutrição e dietoterapia. 9. ed. São Paulo: Roca; 1998. p. 613-28.

16. Shils MR, Olson JA, Shike M. Modern nutrition. In: Mahan LK, Escott-Stump S. Health and disease. 8th ed. Philadelphia: Lea \& Febiger; 1994.

17. Priore SE. Composição corporal e hábitos alimentares de adolescentes: uma contribuição à interpretação dos indicadores de estado nutricional [tese de doutorado]. São Paulo: Escola Paulista de Medicina da Universidade Federal de São Paulo; 1998.

18. Cavadini C. Hábitos alimentares durante a adolescência: contribuição dos lanches. In: Nestlé Nutrition Services. A alimentação da idade pré-escolar até a adolescência. São Paulo: Nestlé; 1995. p. 14-6. (Seminário Nestlé Nutrition, 37).

19. Gambardella AND, Frutuoso MPF, Franchi C. Prática alimentar de adolescentes. Rev Nutr 1999; 12: 55-63.

20. Carlini-Cotrim B, Gazal-Carvalho C, Gouveia N. Comportamentos de saúde entre jovens estudantes das redes pública e privada da área metropolitana do Estado de São Paulo. Rev Saúde Pública 2000; 34: 636-45.

21. Tojo R, Leis R, Pavon P. Necesidades nutricionales en la adolescencia. fatores de riesgo. An Esp Pediatr 1992; 36: (Suppl 49): 80-105.

22. Tershakovec, AM, Kuppler KM, Zemel BS, Katz L, Weinzimer S, Harty MP, Stallings VA. Body composition and metabolic factors in obese children and adolescents. Int J Obes 2003; 27: 19-24.

23. Dietz WH. You are what you eat: what you eat is what you are. J Adolesc Health Care 1990; 11: 76-81.

24. Fisberg M. Obesidade na infância e adolescência. Pediatr Mod 1993; 29: 103-8.

25. Nicholls ES, Caballero B. Las enfermedades crônicas no transmisibles: su prevención em la adolescência. In: Maddaleno M, Munist MM, Serrano CV, Silber TJ, Ojeda EN, Yunes J. La salud del adolescente y del joven. Washington (DC): OPS; 1995. p. 194-202. (Publicación Científica, 552).

26. Marino DD, King JC. Nutritional concerns during adolescence. Pediatr Clin North Am 1980; 27: 125-38.

27. Scagliusi, FB, Lancha Júnior AH. Subnotificação da ingestão energética na avaliação do consumo alimentar. Rev Nutr 2003; 16: 471-82.

28. Huang Y, Song Wo, Schemmel Ra, Hoerr S. What do college students eat? Food selection and meal pattern. Nutr Res 1994; 14: 1143-53.

29. Priore SE. Perfil nutricional de adolescentes do sexo masculino residentes em favela. São Carlos: EDUSFSCAR; 1996

Recebido em 31 de março de 2003

Versão final apresentada em 10 de novembro de 2004

Aprovado em 13 de dezembro de 2004 